\title{
MASTERING BEAM LOSSES AND AGEING ISSUES AT THE ESRF
}

\author{
J.M.Filhol, L Hardy, U. Weinrich \\ ESRF, Grenoble, France
}

\section{Abstract}

The ESRF accelerators have been in full routine operation for more than four years. The source delivers nearly 5600 hours of X-ray beam to 40 beam lines simultaneously, which in turn perform about 700 experiments (or 70.000 hours of experimental work) per year. To maintain free access in the experimental hall only public dose rates are allowed.

However, with the installation of small aperture vacuum vessels in straight sections a dramatic increase of bremsstrahlung outside the corresponding optic hutches was experienced. Hence, thorough studies to understand and master beam losses have been undertaken. This required the development of a beam loss detection system and the adoption of the strategy of using scrapers for beam collimating.

In addition, great care is taken to detect the first traces of ageing of equipment (for example due to irradiation) and therefore avoid, as far as possible, failures. It is indeed true to say that the more failures there are, the more the beam losses and the more the dose level!

\section{BEAM LOSSES}

\section{BEAM LOSS MECHANISMS}

The electron beam losses can be sorted into three major categories: beam losses during injection, electron losses during stable stored beam due to the finite lifetime and total beam losses following equipment failures or voluntary beam kills.

- Injection losses occur when the injected electrons do not fall within the longitudinal or transverse acceptance. At a typical injection efficiency of $80 \%$ they are responsible for $20 \%$ of the losses. Given the energy acceptance of the storage ring of more than $3 \%$ most of the injection losses were measured to be due to transverse mismatching of the injected beam.

- Among the different contributions to the lifetime limitation only four effects appeared to be relevant: Elastic gas scattering, inelastic gas scattering, Touschek scattering and Compton scattering. In few bunch mode delivery the Touschek lifetime dominates, by far, the losses whereas during the standard multibunch filling pattern all four contributions remain within the same range. The Compton scattering of photons on the electrons only takes place if the GRAAL experiment (which shoots with a laser on the beam) is in operation. The beam line is allowed to reduce the beam lifetime by no more than $20 \%$.

- Other major losses are experienced due to voluntary beam kill with a scraper jaw or due to equipment failure.

Only the lifetime losses and the equipment failures contribute to radiation problems around the optic hutches in user service mode whereas all losses are responsible for the chamber activation and radiation damage inside the tunnel.

\section{BEAM LOSS POSITIONS}

\subsection{The beam loss detector system}

To detect the losses a set of beam loss detectors were installed around the ring. The detection principle is based on the creation of light in a scintillator material when a high-energy particle crosses the detector volume. To suppress synchrotron radiation background the scintillator material is placed inside a protection of lead. Experience has shown that the detector readings are not very reliable due to too high synchrotron radiation background and damage caused during interventions. In addition, their positioning at the entrance of the ID vessels did not allow the comparison of the losses from different cells due to the different chamber and location configurations. An upgraded version with improved synchrotron radiation shielding was therefore installed and tested. The detectors are placed on the tunnel wall close to end of the straight section. First results show that the synchrotron radiation background is suppressed and that, in the case of loss changes, the signals of the detectors act correspondingly.

\subsection{Tune shift with amplitude}

Beam losses due to Touschek scattering were found to occur in the vertical plane. An explanation was found upon investigation of the tune shift with energy. Electrons 
with $3.5 \%$ energy reduction find their vertical tune reduced from the nominal 11.39 to 11.00 . Given that the integer resonance kills the beam, scattering effects with energy losses finally lead to a vertical oscillation.

This tune shift with amplitude effect was found to be the energy acceptance limiting factor. Consequently the majority of electron losses ended up in losses on Insertion Device chambers.

\subsection{Scraping effect of a single chamber.}

A vacuum chamber of the length $2 \mathrm{D}$ and half aperture $\mathrm{d}$ has, for a minimum beta function $\beta$ in the middle of the chamber, a total acceptance of $A=\left(d^{2} \cdot \beta\right) /\left(\beta^{2}+D^{2}\right)$. If the beta function is equal to half of the length of the vacuum vessel the total acceptance becomes maximum.

\subsection{Interactions on chamber walls}

Simulations were made to investigate the collision of the electrons on a chamber wall. One important result was that if the impact takes place with a rather small angle a large part of the electrons will be slightly scattered and will only loose a small fraction of their energy [1]. These electrons have the potential of being lost downstream. This effect was measured when closing a scraper jaw.

\section{LOSS REDUCTION MEASURES}

-Low beta optics: One major change towards reduced losses was the switch to an optics with $2.5 \mathrm{~m}$ vertical beta function in the middle of the straight section. This is the optimum value in terms of vertical acceptance for the $5 \mathrm{~m}$ long vessels. This directly increased the vertical elastic gas scattering lifetime and the acceptance for vertical missteering during injection.

- The main filling pattern was changed from a $1 / 3$ filling (350 bunches) to a $2 / 3$ filling (700 bunch) thus doubling the Touschek lifetime.

- The septum sheet was placed further away from the beam $(19.5 \mathrm{~mm}$ instead of $13.5 \mathrm{~mm})$. This increased the transverse acceptance and therefore the Touschek and inelastic gas scattering lifetime.

-For any operating mode, the refill consists of a toppingup and does not require to kill the stored beam.

- Limitation on injected beam: In order to comply with the maximum authorised dose rate in the experimental hall, and based on the radiation measured on the roof of the tunnel in case of localised loss, the amount of injected electrons into the storage ring was limited to $12 \mu \mathrm{C}$ per four hour period.

\section{PROTECTION WITH SCRAPERS}

Closing vertical jaws proved to be very efficient in removing the large majority of losses from the low aperture insertion device vessels. However, closing horizontal jaws also proved to help because this localises the losses due to the transverse acceptance limitation. A strategy was adapted which consists in closing all available scraper jaws to a position which hardly reduces the lifetime during stable stored beam. During injection the scraper jaws are closed even further to the limit of reducing the injection efficiency.

A new design for scrapers with appropriate shielding is being developed.

\section{AGEING ISSUES \& MAINTENANCE}

\subsection{Introduction}

For a source which delivers 5600 hours per year of X-ray beam to 40 beamlines, the availability and the Main Time Between Failures (MTBF) must be as high as possible, which is a major challenge. We can assess that these goals are achieved at the ESRF since the availability in 1998 reached $95.3 \%$ for a MTBF of 31 hours. In the remaining $4.7 \%$, only $3.7 \%$ is due to faults and $1 \%$ represents the dead time due to refills. Great care is therefore necessary to maintain this good performance at its present level, requiring significant preventive maintenance.

In addition, as explained above, a failure of equipment is synonymous to a beam kill and hence participates in the generation of radiation. This is also a good incentive to optimise the preventive maintenance strategy.

\subsection{Preventive and curative maintenance}

We can classify the failures into two types: those that last a long time (affecting the beam availability of the Machine) and those which are repetitive (with a greater effect on the MTBF). For this second class of failures, which create most of the doses due to 'beam kills', priority is given to our strategy of preventive actions.

- The first system concerned is the Radio frequency equipment ( $40 \%$ of the total down time). As a preliminary step, a third RF unit (including a klystron and a pair of cavities) was added in the Storage Ring. In normal operation, two klystrons feed six cavities. This configuration, which is oversized with respect to our needs, enables the operation of each klystron below its maximum capacity and allows to distribute the power to six cavity couplers instead of four. Since then, the 'RF crowbars' which were the first cause of beam failures when operating close to the limits on both klystron and cavities, have completely disappeared. At the same time, more protective interlocks ( $\mathrm{RF}$ arc detections) were integrated into the RF equipment, which on the one hand reduced the possibility of major failures, but on the other increased the frequency of beam trips. Fine tuning of the threshold of these interlocks had to be done to suppress wrong triggering. Finally the upgrading of the RF control 
system, with fast data logging, proved to be very helpful in tracing the origin of the trips.

- Another source of frequent trips came from the vacuum controllers which were reading wrong values from vacuum gauges, triggering the closure of sector valves. Weaknesses in the electronic design were found which were enhanced by a non optimised shielding of the tunnel cables against the propagation of electromagnetic perturbations. Since then, all gauge controllers have been replaced and indeed, for one year now, the Machine has not been perturbed by problems of this type. Moreover, a systematic modification of the cables arrangement inside the tunnel which aims at reinforcing the shielding against EMC perturbations has begun.

\subsection{Ageing issues}

Most of the equipment is now eight years old and ageing effects have started to become apparent.

-Several small vacuum leaks have started to appear since the end of 1997. The cause for these leaks was found to be a very slow corrosion process developing in the thickness of the stainless steel very likely caused by a welding agent which was badly removed at the time of manufacture of these vacuum vessels. Some of these leaks were temporarily fixed with "varnish" and a batch of spare vessels was ordered.

- A lot of blockage problems have been encountered with the various water cooling circuits and reduced flow rates have frequently been experienced. The latter have been recovered either by reversing the flow direction or by flushing the pipes with acid, which was particularly necessary on the small diameter pipes. We are currently carrying out an analysis of the small particles present in the water circuit which are responsible for the blockages.

- A complete review of the spare parts has been undertaken for all equipment and spares have been ordered for all critical items. Amongst others, this concerns electronic power components which appeared to have become obsolete and the procurement of which is time consuming.

\subsection{Radiation damages}

On a light source, radiation damages can be induced either by "conventional radiation", generated by the lost electrons (cascade) or by the intense X-rays which are emitted (or scattered) essentially on the beam axis plane. The crotch area (downstream the bending magnet) is the area were the highest X-ray dose is generated.

Since the first beam in 1992, about 4000 Amp.hours of electron beam current have been integrated and are producing synchrotron radiation $(0.1$ to $100 \mathrm{KeV})$, part of which hits equipment in the tunnels. During the first years of the Machine, some lead protection was progressively installed to shield some sensitive equipment located close to the medium plane (BPM/RF multiplexers, insertion devices optical encoders, ..). However, in 1998, the first disturbing effects associated with radiation damages really began to appear :

- Crazy readings of vacuum pressure levels: Several cables of Penning gauges were seriously toughened due to radiation and their insulation sheaths no longer performed their function. Since then, several of these cables have been found damaged and have been replaced. A systematic check is now done at every Machine shutdown. Furthermore, other cables driving low level signals such as magnet interlock have also been found damaged in areas close to the beam.

- On this occasion, we also found that most of the insulating sheaths of the sextupoles conductors had started to become brittle. Those which were more heavily damaged were immediately covered with a radiationproof sheath. As a measure of preventive maintenance, all of the similar conductors have been re-insulated.

- Rather more serious problems occurred with the water flexible hoses. Guarantied to be "radiation resistant" most of them did not present any externally visible signs of ageing due to radiation. However, one after the other several of them tore (creating five water leaks in less than one year). Indeed, we discovered that the inner part of the hose had turned brown and had hardened. Once again, all the hoses that were most exposed were immediately replaced with another type of "radiation-proof" water pipe. This costly operation ( 12\$/meter for the pipe) represented a workload of 1200 man-hours.

\section{SUMMARY}

The analysis of the loss mechanisms lead to the conclusion that the majority of losses take place on the small aperture insertion device chambers. Closing scraper jaws was found to be efficient in concentrating the losses on less critical points. Furthermore several improvements enabled to reduce the amount of losses. Following the success of the actions taken, half of the straight sections are now operated with $11-\mathrm{mm}$ inner vertical aperture vessels without problems. In addition, several 8-mm inner vertical aperture vessels are now installed and in their test phase. In order to limit the number of beam kills due to failures, several preventive and curative actions took place. Following the first effects of irradiation on some material located in the tunnels in 1998, actions were quickly undertaken to resolve these problems. We expect to see the first beneficial effects in 1999 .

[1] Behaviour of the beam in incident collisions with the scraper, $T$. Günzel, ESRF technical note 10-96 Theory, July 1996 